\title{
FONDAMENTO ETICO DEI DIRITTI UMANI E IMMIGRAZIONE
}

\section{ETHICAL FOUNDATION OF HUMAN RIGHTS AND IMMIGRATION}

\author{
Alfredo Rodrigues ${ }^{*}$
}

\section{RIASSUNTO}

Dopo l'11 settembre 2001 l'agenda politica dei governi dei tradizionali paesi di immigrazione tende a introdurre misure sempre più severe contro immigrati non autorizzati e richiedenti asilo. Ma, l'inasprimento delle norme e l'atteggiamento generale di chiusura dei governi si scontrano con i paletti posti dal sistema giudiziario in nome dei diritti umani. L'orizzonte di senso dei diritti umani esige che lo Stato riconosca che anche i migranti in situazioni irregolari, hanno diritti che devono essere rispettati: in primis il diritto alla vita. Ciò deriva dal fatto che $\mathrm{i}$ diritti umani sono universali (valgono per ogni uomo), fondamentali (proteggono non tutte le fonti e condizioni possibili e immaginabili del benessere, ma solo gli interessi e i bisogni fondamentali dell'uomo). Il presente articolo ha lo scopo di indagare il fondamento etico dei diritti umani e il loro ruolo nella protezione dei migranti.

Parole chiave: Fondazione, diritti umani, autonomia, riconoscimento

\begin{abstract}
After 11 September 2001, the political agenda of the governments of traditional immigration countries tends to introduce ever stricter measures against unauthorized immigrants and asylum seekers. But, the tightening of the rules and the general attitude of closing governments clash with the stakes placed by the judicial system in the name of human rights. The horizon of meaning of human rights requires that the State recognizes that even migrants in irregular situations have rights that must be respected: first and foremost the right to life. This derives from the fact that human rights are universal (they are valid for every man), fundamental (they protect not all the possible and imaginable sources and conditions of wellbeing, but only the fundamental interests and needs of man). This article aims to investigate the ethical foundation of human rights and their role in the protection of migrants.
\end{abstract}

Keywords: Foundation, human rights, autonomy, recognition

"Università di Roma (Italia) "La Sapienza" 


\section{INTRODUZIONE}

Le teorie sulla giustificabilità delle norme morali in generale, così come quelle sulla giustificabilità dei diritti umani, si possono classificare in diversi modi. In prima battuta, si può distinguere tra gli approcci che contestano radicalmente la possibilità di una fondazione e approcci che, invece, ritengono possibile rivenirla. Uno scetticismo radicale definito dalla negazione sostanziale della giustificabilità delle norme morali può radicarsi nelle forme dell'emotivismo, del decisionismo, del soggettivismo, del relativismo, del naturalismo o del decostruzionismo. Il non- scetticismo sostiene, al contrario, che sia possibile addurre ragioni per i diritti umani, che possano sollevare una pretesa di oggettività, correttezza e verità.

È possibile individuare l'origine della spaccatura teorica, se non prima, almeno nel momento dell'elaborazione della Dichiarazione universale dei diritti dell'uomo, approvata dall'Assemblea generale delle Nazioni Unite il 10 dicembre $1948^{1}$. In quella occasione la questione del fondamento " $\mathrm{fu}$ accantonato, per non correre il rischio di non approdare alla pubblicazione della Dichiarazione universale dei diritti dell'uomo. Si credette sufficiente un accordo su uno stesso corpo di convinzioni concernenti l'azione" (ALEXY, 2015, p.10; DE PAOLIS, 2014, pp. 3435). Questa posizione fu ritenuta l'unica possibile, tuttora considerata come tale e dalla quale scaturisce

\footnotetext{
${ }^{1}$ Nella storia della formazione delle dichiarazioni dei diritti si possono distinguere almeno tre momenti. Le dichiarazioni nascono come teorie filosofiche. Infatti, è possibile rintracciarle, in nuce, nel pensiero filosofico, anche antico. Se non vogliamo risalire sino all'idea Stoica della società universale degli uomini razionali - il saggio è cittadino non di questa o di quella patria ma del mondo - l'idea che l'uomo in quanto tale ha dei diritti per natura che nessuno, neppure lo Stato, gli può sottrare e che egli stesso non può alienare è stata elaborata dal giusnaturalismo moderno, il cui rappresentante è John Locke. Per Locke il vero Stato dell'uomo non è lo Stato civile ma quello naturale, cioè lo stato di natura in cui gli uomini sono liberi ed eguali, e lo Stato civile è una creazione artificiale che non ha altro scopo che quello di permettere la più ampia esplicazione della libertà e dell'uguaglianza naturale. Non a caso, l'incipit della Dichiarazione universale afferma: "gli uomini nascono liberi ed eguali in dignità e diritti". Nella seconda fase, le teorie filosofiche vengono accolte dai legislatori. Accade con le Dichiarazioni degli Stati americani e della Rivoluzione francese. Si assiste alla formazione dei diritti positivi, così il secondo momento della storia della Dichiarazione dei diritti umani consiste nel passaggio dalla teoria alla pratica, dal diritto solennemente pensato al diritto attuato entro i confini nazionali. I diritti dell'uomo sono tali solo in quanto diritti del cittadino di questo o quello Stato particolare. Il 10 dicembre 1948 inizia la terza e ultima fase in cui l'affermazione dei diritti è insieme universale e positiva: universale nel senso che i destinatari dei diritti non sono più soltanto i cittadini di un determinato Stato ma tutti gli uomini; positiva nel senso che i diritti dell'uomo devono essere proclamati ed effettivamente protetti. Dunque i diritti dell'uomo nascono come diritti naturali universali, si svolgono come diritti positivi particolari e poi come diritti positivi universali.
}

l'inevitabile esito per cui i diritti umani sono pensati come il risultato di un accordo pragmatico, di una convenzione, espressa dalla volontà politica storicamente determinata.

Come è possibile parlare di un consenso generale su alcuni valori, quando di fatto non si riesce a trovare una giustificazione generale di essi? L'idea che ogni persona, in qualsiasi parte del mondo, indipendentemente dalla nazionalità, dal luogo di residenza, dalla razza, dal sesso, dalla casta o dalla comunità di appartenenza, abbia alcuni diritti fondamentali che gli altri siano tenuti a rispettare ha bisogno di essere fondata teoreticamente, non soltanto affermata, perché i diritti umani in quanto tali hanno una validità morale e "un diritto vale moralmente se può essere giustificato di fronte a ogni individuo che prende parte ad una fondazione razionale"2 (ALEXY, 2015, p.9; BOBBIO, 1990, p.262).

Non è difficile capire che, per chi lotta per i diritti umani, cercare giustificazioni concettuali, magari per convincere qualche teorico scettico, è poco rilevante, data l'evidente urgenza di reagire ai terribili soprusi che ci sono nel mondo. Ciò attesta che i diritti umani sono fini meritevoli di essere perseguiti, ma che nonostante la loro desiderabilità, non sono ancora stati tutti, dappertutto, e in eguale misura, riconosciuti. Pertanto, la giustificazione dei diritti compresi nella Dichiarazione universale, può contribuire a una adesione consapevole e risoluta. Nelle pagine successive la nostra attenzione sarà rivolta in un primo momento nell'individuazione del fondamento etico di quei diritti che valgono in ogni situazione e per tutti gli uomini indiscriminatamente. In un secondo momento esamineremo l'efficienza dei diritti umani nella protezione dei migranti.

\footnotetext{
${ }^{2}$ La validità morale dei diritti umani coincide con la loro esistenza che consiste nella loro giustificabilità. La validità morale dei diritti umani include anche il quadro giuridico-positivo. Il Patto Internazionale sui Diritti civili e politici del 19 dicembre 1966 ne è l'ottimo esempio. Per secoli si è ritenuto per nulla naturale che le donne andassero a votare. Oggi non è così. Tuttavia, tale positivizzazione non può essere intesa in modo statico, bensì inserita in un ordine dinamico. I diritti umani non sono stati dati tutti in una volta e neppure congiuntamente. I diritti umani, in quanto diritti morali, non possono essere resi inefficaci da norme giuridico-positive, essi sono il criterio a cui ogni interpretazione di ciò che è positivamente statuito deve conformarsi. Può avvenire che una decisione presa in un tribunale sia contraria ai diritti umani.
} 


\section{ALLA RICERCA DEL FONDAMENTO ETICO DEI DIRITTI UMANI}

Norberto Bobbio nella sua opera L'età dei diritti sostiene che la questione del fondamento assoluto dei diritti umani "è soltanto un'illusione" (BOBBIO, 1990, p.14). I diritti umani sono valori ultimi, in quanto tali non si giustificano, si assumono: "ciò che è ultimo, proprio perché è ultimo, non ha alcun fondamento" (BOBBIO, 1990, p.8). Contro coloro che contestano la possibilità di una fondazione, sono stati avanzati diversi approcci che invece la sostengono. Il primo e il più noto orizzonte di senso della fondazione dei diritti umani è di carattere religioso. Partiamo con la considerazione dell'Alto Commissariato delle Nazioni Unite per i diritti dell'uomo, Mary Robinson. Nel suo articolo I diritti dell'uomo, storia e speranza, scrive:

Sono convinto che le radici più profonde di ciò che chiamiamo ora i diritti dell'uomo siano situate un po' al di là di noi al disopra di noi: ad un livello più profondo del mondo delle convinzioni umane, in un campo che definirei, per semplicità, metafisico. Anche se non sempre lo realizzano, gli uomini - le sole creature pienamente coscienti della loro esistenza e della loro mortalità, che percepiscono il loro ambiente circostante come un mondo e hanno una relazione interiore con questo mondo - traggono il loro senso di dignità e di responsabilità da questo mondo percepito come un tutt'uno. I cristiani lo dicono in modo molto semplice: a quel livello, l'uomo è l'immagine di Dio (ROBINSON, 2016, p.24).

"L'uomo è l'immagine di Dio": sono le parole che troviamo nelle pagine iniziali del primo libro della Sacra Scrittura, che descrivono la creazione dell'uomo e della donna (GEN. 1, 26-27). Dire che "Dio è creatore" non significa esprimere solo una convinzione teorica, ma anche cogliere l'orizzonte originario dell'agire gratuito e misericordioso di Dio a favore dell'uomo. Dio liberamente dà l'essere e la vita a tutto ciò che esiste. L'uomo e la donna, creati a Sua immagine e somiglianza, sono chiamati ad essere il segno visibile e lo strumento efficace della gratuità divina. Pertanto, essendo ad immagine di Dio, l'individuo umano ha la dignità di persona; non è soltanto qualcosa, ma qualcuno. È capace di conoscersi, di comprendersi, di liberamente donarsi e di entrare in comunione con altre persone; è chiamato per grazia, ad un'alleanza con il suo Creatore (homo est Dei capax), a dargli una risposta di fede e di amore che nessun altro può dare in sua sostituzione. (PONTIFICIO CONSIGLIO DELLA GIUSTIZIA E DELLA PACE, Compendio della dottrina sociale della Chiesa, 2004, n. 108; FRANCESCHINI, 2012, p.139).

La validità dell'argomento religioso presenta uno svantaggio insito perché è tale solo per coloro che credono in Dio e nell'immagine divina dell'uomo. L'esatto opposto dell'approccio religioso è l'orientamento biologico o sociologico. Secondo questa linea di pensiero la morale è una forma di altruismo; è possibile osservare un comportamento altruistico "per la sopravvivenza di patrimonio genetico di un individuo", in modo particolare nella cura dei propri figli e nel sostegno parentale, ma vale anche per quelle forme di reciproco altruismo che conduce all'aiuto vicendevole (ALEXY, 2015, p.11).

Anche questo argomento presenta una debolezza: è vero che la tendenza a massimizzare il proprio successo riproduttivo può generare, in una serie di casi, il rispetto e l'aiuto verso ogni persona. Ma si tratta soltanto di un modello comportamentale, connesso con l'indifferenza verso coloro i quali non rientrano nella dinamica dello scambio altruistico, nel nostro caso gli immigrati. Se i diritti umani sono suscettibili di fondazione, pertanto, ciò deve avvenire non attraverso l'osservazione della natura biologica dell'uomo, ma attraverso la spiegazione della sua essenza culturale.

Il terzo approccio depone a favore dell'intuizioni. Il modello intuizionistico sostiene che i diritti umani siano giustificati perché la loro esistenza o validità è evidente. Ma l'evidenza dei valori proclamati tali non regge al peso della verifica storica: sotto la lente della Storia, l'evidenza dei valori si fa mutevole e storicamente determinata dal momento dato. Oggi è evidente che non si debbano torturare i detenuti, eppure per molti secoli la tortura fu accettata e difesa come una normale procedura giudiziaria. Possiamo dire ancora di più: dopo gli attacchi terroristici dell'11 settembre 2001 a New York, a Londra nel 2005 o a Mumbai nel 2008 e via dicendo, si può ritenere ragionevole l'idea di trattare come materia di diritti umani l'eliminazione della paura del terrorismo, anche qualora i timori andassero al di là di quanto giustificato dalle probabilità statistiche.

Questo esempio dimostra che i diritti elencati nella Dichiarazione universale non sono i soli e possibili diritti dell'uomo - sono i diritti dell'uomo storico quale si configurava alla mente dei redattori 
dopo la tragedia della Seconda guerra mondiale. Non occorre molta immaginazione per prevedere che lo sviluppo della tecnica, la trasformazione di condizioni economiche e sociali, l'ampliamento di conoscenze e l'intensificazione dei mezzi di comunicazione potranno creare occasioni favorevoli alla nascita di nuovi bisogni e quindi a nuove richieste di libertà $\mathrm{e}$ di poteri (BOBBIO, 1990, p. 28).

Uno sguardo diverso sulla fondazione dei diritti umani concerne l'universalismo consensuale. Secondo questo punto di vista un valore è tanto più fondato quanto più è consentito. In altri termini, l'universalismo consensuale consiste nell'idea che la stipulazione giuridica dei diritti fondamentali, quali diritti universali, supponga la tesi assertiva che essi siano valori oggettivi e/o universalmente condivisi. Così i diritti contenuti nella Dichiarazione universale sono fondati perché il Documento rappresenta nella storia dell'umanità un sistema di principi fondamentali della condotta umana liberamente ed espressamente accettato (almeno teoricamente) da "tutti cittadini del mondo", attraverso i loro rispettivi governi.

[...] oggi il problema del fondamento dei diritti dell'uomo ha avuto la sua soluzione nella Dichiarazione universale dei diritti dell'uomo, approvata dall'Assemblea generale delle Nazioni Unite il 10 dicembre 1948. La Dichiarazione universale dei diritti dell'uomo rappresenta la manifestazione dell'unica prova con cui un sistema di valori può essere considerato umanamente fondato e quindi riconosciuto: e questa prova è il consenso generale circa la sua validità. I giusnaturalisti avrebbero parlato di consensus omnium gentium o humani generis (BOBBIO, 1990, p.18-19).

Se la maggior parte dei governi esistenti hanno proclamato solennemente che i diritti umani sono fini desiderabili, è segno che hanno trovato buone ragioni per farlo, anche se ciò non garantisce 1'attuazione dei diritti proclamati. Per Luigi Ferrajoli questa tesi "è empiricamente falsa" (FERRAJOLI, 2018 , p. 24). Il consenso non è nient'altro che un accordo di convenzioni. Se un accordo sui diritti umani esistesse, si potrebbero richiedere ragioni in relazione a convinzioni convergenti. Se, però, il consenso non viene manifestato da tutti individui, allora sono indispensabili gli argomenti. Quale sia la giustificazione, la sua validità dipende esclusivamente dal consenso delle semplici maggioranze.
I diritti presenti nella Dichiarazione universale - il diritto alla libertà di coscienza, il diritto alla libertà di movimento e via dicendo - "non sono affatto da tutti condivisi" (ivi). Non lo sono, non solo da gran parte delle persone di cultura diversa da quella occidentale, ma neppure da molti di coloro che pure appartengono per nascita alla cultura occidentale stessa. Se i diritti contenuti nella Dichiarazione universale fossero messi ai voti, ci sarebbero i diritti che non otterrebbero nemmeno il 10\% dei consensi. Luigi Ferrajoli parla di $1 \%$. La tesi assertiva della condivisione universale dei diritti è perciò empiricamente falsa.

Una quinta proposta sul carattere fondativo dei diritti umani è di impronta strumentale. Secondo questa pista teorica, il riconoscimento dei diritti umani è necessario se si vuole massimizzare la propria utilità individuale. Robert Alexy compendia tale posizione in questi termini: "se tu non vuoi essere ucciso, allora devi rispettare il suo diritto alla vita" (ALEXY, 2015, p. 12), asserzione nella quale si avverte l'eco hobbesiana della fondazione dello Stato civile. Lo Stato hobbesiano è il risultato di un patto nato dalla paura di una morte violenta. L'aggressione, reale o possibile nello stato di natura, genera prima la paura, poi l'impulso a sottrarvisi attraverso un accordo basato sulla rinuncia di ogni individuo di tutti i diritti naturali, tranne del diritto alla conservazione della vita (HOBBES, 1989; SCHINO, 2016, p. 82-83).

Anche se non totalmente inutile, l'approccio strumentale non fonda i diritti umani: lo testimonia la Storia e l'esistenza di soggetti che massimizzano la loro utilità (anche a lungo termine) mediante lesione dei diritti umani di altre persone.

Alle falle individuate dalle visioni teoriche precedentemente menzionate, si può contrapporre lo sguardo del pensiero teorico discorsivo di Habermas (HABERMAS, 1989; 2013). Si tratta della cosiddetta fondazione esplicativa e consiste nel rendere esplicito ciò che di necessariamente implicito è contenuto nella prassi umana. Si assume che sia corretta la tesi secondo cui la libertà e l'uguaglianza dei partecipanti alla discussione, in quanto partecipanti, sia un presupposto che non può essere evitato se si entra nell'ambito dell'argomentazione. Afferma Stefano Petrucciani nell'introduzione dell'opera di Guido Calogero dal titolo Filosofia del dialogo: "chi infatti volesse convincerci ad abbandonare la volontà di dialogare con gli altri dovrebbe, a questo fine, instaurare con 
noi un dialogo, e quindi si collocherebbe proprio su quel terreno che vuole convincerci ad abbandonare" (PETRUCCIANI, 2015, p. III).

Questo argomento non può essere universalizzato perché sarebbe arbitrario estenderlo a tutte le comunità politiche - per esempio, uno Stato teocratico - che non condividono i presupposti discorsivi o che non hanno la fiducia nell'argomentazione razionale come strumento di risoluzione dei conflitti d'azione. La giustificazione discorsiva dei diritti soggettivi di Habermas possiede valore solo all'interno di un contesto nel quale possano essere fatte valere le assunzioni che legano la validità delle norme al contesto dei diretti interessati ${ }^{3}$.

Il tentativo habermasiano di fondare $\mathrm{i}$ diritti umani attraverso un argomento pragmaticotrascendentale, cercando di mostrare che non è razionalmente possibile non accettare i principi della razionalità comunicativa è problematico, dal momento che per apprezzare la forza della critica bisogna già, in un certo modo, trovarsi all'interno di una comunità che aderisce almeno in parte a certi principi di razionalità. Habermas era anche consapevole di questa difficoltà: per esempio, quando afferma che "il discorso interculturale intorno ai diritti non può progredire in maniera decisiva finché tutti i partecipanti non avranno imparato a guardare le proprie tradizioni con gli occhi dei propri interlocutori e non si mostreranno disposti ad apprendere dalle loro visioni del mondo" (MARCHETTONI, 2012, p.7).

In questa visuale, il piano del discorso non equivale però all'ordine dell'azione. La necessità di trattare l'altro nel discorso come parimenti legittimo, non implica che sia necessario riconoscerlo come libero e uguale anche nell' ambito dell'agire: del resto anche con gli schiavi si può intavolare un dialogo. Se si vuol passare dalla libertà e uguaglianza del mondo etereo del discorso alla libertà e uguaglianza nello spazio angusto dell'azione occorrono due concetti distinti ma profondamente connessi. Il primo è il concetto di partecipazione sincera al discorso; il secondo, invece,

\footnotetext{
${ }^{3}$ Il tentativo habermasiano di fondare i diritti umani attraverso un argomento pragmatico-trascendentale, cercando di mostrare che non è razionalmente possibile non accettare i principi della razionalità comunicativa è problematico, dal momento che per apprezzare la forza della critica bisogna già, in un certo modo, trovarsi all'interno di una comunità che aderisce almeno in parte a certi principi di razionalità. Habermas era anche consapevole di questa difficoltà: per esempio, quando afferma che il discorso interculturale intorno ai diritti non può progredire in maniera decisiva finché tutti i partecipanti non avranno imparato a guardare le proprie tradizioni con gli occhi dei propri interlocutori e non si mostreranno disposti ad apprendere dalle loro visioni del mondo.
}

è il concetto dell'autonomia (ALEXY, 2015, p. 15). Autonomo è chi agisce secondo regole e principi che egli giudica corretti dopo sufficiente ponderazione. Prende sinceramente parte a discorsi morali chi vuol risolvere i conflitti sociali mediante consensi prodotti e controllati discorsivamente.

Il partecipante al discorso sincero e genuino, secondo Robert Alexy, "connette [...] le sue capacità discorsive con l'interesse a far uso di esse nell'azione" (ivi). Questa connessione tra capacità e interesse implica il riconoscimento dell'altro come autonomo. Chi riconosce l'altro come autonomo, lo riconosce come persona. Chi lo riconosce come persona, gli attribuisce dignità. Chi gli attribuisce dignità, riconosce i suoi diritti umani. Ma che cos'è la dignità umana? Habermas nel suo brevissimo saggio dal titolo Il concetto della dignità umana e l'utopia realistica dei diritti umani sulla scia di Kant, definisce la dignità umana come "il rispetto per sé e il riconoscimento sociale nello spazio e nel tempo" (HABERMAS, 2012, p.18). Si tratta del riconoscimento reciproco, incondizionato, laddove ogni persona rispetta se stesso e gli altri, non come semplice mezzo ma sempre come un fine. Per chiarire bene questo concetto passiamo al paragrafo successivo.

\section{IL RICONOSCIMENTO DELL'ALTRO COME AUTONOMO E IL DIRITTO}

Il precursore della tematica del riconoscimento è storicamente considerato Hegel, il quale, nella Fenomenologia dello spirito, ponendosi contro la concezione kantiana della coscienza considerata solipsistica, mostra come sia importante per l'uomo che vive in società il riconoscimento da parte degli altri, riconoscimento senza il quale la propria soggettività non può svilupparsi (HEGEL, 2000, pp.275-291). Per Kant e per l'illuminismo "la libertà individuale è un diritto primario, perché essendo l'uomo un essere razionale, è in quanto tale indipendente dal contesto in cui esercita la sua libertà" (MURA, 2008, p.170). Hegel, invece, individua una crepa nella nozione, propria dell'illuminismo kantiano, di libertà dell'individuo, considerata astratta, perché sostenitrice di una sussistenza a priori della libertà dell'individuo; senza il pieno riconoscimento da parte dell'altro, essa non sarà mai reale.

Paul Ricoeur, nel suo ultimo libro dal titolo significativo, Percorsi del riconoscimento (2005), 
si sofferma a lungo sulla nozione di riconoscimento (Anerkennung), ritenendo ancora valida la critica hegeliana alla nozione astratta di libertà dell'individuo. Il riconoscimento è autentico solo se è "effettivo" ovvero se è capace di superare la dialettica servopadrone propria della metafora hegeliana.

La nostra esperienza comune ci fa "toccare con mano" la caratteristica peculiare della nostra condizione umana, che è quella di essere un uomo che non assomiglia a "nessun altro", in quanto unico, irripetibile, per la propria esperienza individuale di vita non paragonabile a nessun'altra; un essere particolare che assomiglia ad "alcuni altri", che condividono con lui determinate appartenenze sociali, identità linguistiche-culturali, religiose, etiche ereditate per nascita e/o costituite per scelta volontarie; e un essere che assomiglia a "tutti gli altri", per la sua appartenenza alla comune natura umana, che gli conferisce la sua dignità radicale. Se questo è 1'uomo, "il diritto di essere quest'uomo"4 (PEROTTI, 2004, p.50) implica il diritto all'indivisibilità e all'interdipendenza di queste tre dimensioni costitutive della sua identità globale: “l'unicità, la pluralità, l'universalità e riconoscere che ogni diritto umano è un'interfaccia tra il particolare e l'universale: la realizzazione di un singolare" (PEROTTI, 2004, p. 49).

Riconoscere l'altro come singolarità, unica, irripetibile, portatrice di una sua struttura intersoggettiva trascendentale, da cui può trarre i valori morali di una vita buona dai buoni rapporti intersoggettivi che in essa sa stabilire, significa riconoscere i diritti inalienabili, irrinunciabili e non negoziabili della persona umana. La relazione di riconoscimento reciproco tra le soggettività che compongono un contesto sociale è dunque fondata sia nella personalità dell'altro, e dei suoi diritti, sia nella propria soggettività e nei propri diritti. Se l'uomo non fosse un essere in relazione - con se stesso, con gli altri, con la natura e con Dio - non sarebbe neppure soggetto di diritto. Ma che cos'è il diritto?

Chiedersi cos'è il diritto può suscitare una reazione scettica o quantomeno annoiata,

\footnotetext{
${ }^{4}$ Abitualmente noi parliamo di "diritti umani" al plurale. Con questa espressione noi intendiamo riferirci a quei diritti che possono aspirare ad essere universalmente accettabili, anche al di fuori delle loro origini culturali (Occidente) ed esprimere così un accordo tendenzialmente universale su un insieme minimo di valori, variamente ruotante intorno allidea della dignità umana. Questa declinazione al plurale è tradotta al singolare da Antonio Perotti con l'espressione: il diritto di essere quest'uomo. Si riferisce all'uomo contestualizzato, alla sua esperienza individuale e collettiva dell'umanità.
}

nell'impossibilità di pervenire a una risposta convincente e condivisa, dovendoci accontentare di elenchi più o meno lunghi di significati del diritto quali giustizia, persona, relazione, legge naturale, legge positiva, ecc., che al massimo consentono di cogliere la complessità del fenomeno. La concezione classica del diritto è essenzialmente "ciò che è giusto, ossia l'oggetto della giustizia quale virtù che muove a dare a ciascuno il suo diritto" (JOSÈ ERRÀZURIZ , 2017, p. 263). Il diritto prima di essere legge o norma, prima di essere facoltà o diritto soggettivo, o potere di fare o non fare, è anzitutto un corredo o un patrimonio che ogni persona possiede per il fatto stesso di essere persona, perché si possa realizzare e attuare una propria identità.

Affermare il diritto come una facoltà soggettiva significa affermare il primato della persona. La rivendicazione di questo primato rivela a sua volta la fondazione nella stessa persona del diritto. "I diritti sono prima di tutto gli strumenti di cui la persona dispone per il semplice fatto che è persona, perché essa possa orientarsi nella vita. I diritti sono gli strumenti che l'uomo ha per il cammino della sua libertà; ma non sono creati dalla sua libertà in modo arbitrario" (DE PAOLIS, 2014, p. 39). Se il diritto viene prima della legge e prima dell'autorità, i diritti umani sono giustificabili in quanto per-esistono, meta-positivamente, alla volontà politica, essendo connaturati all'uomo.

La persona è origine e fine dello stesso ordinamento giuridico. Da questa prospettiva, i diritti umani si possono dire sufficientemente fondati solo se hanno il loro fondamento nella persona umana. Proprio per questa ragione, essi hanno anche il loro fondamento etico, perché la persona, in quanto essere responsabile, deve agire nella moralità ovvero eticamente; deve misurare moralmente la sua azione, sia in relazione al suo stesso essere che in relazione agli altri che godono della sua stessa dignità e meritano pertanto un rispetto. Il rispetto della dignità umana non può prescindere dal rispetto del prossimo, nessuno escluso, come "altro se stesso", tenendo conto prima di tutto della sua vita e dei mezzi necessari per viverla degnamente (FRANCESCHINI, 2012, p. 138). Il rispetto dell'alterità, quando quest'ultima si trova in situazione di disuguaglianza, esige che io non solo riconosca la "differenza", ma che operi per colmare la carenza di uguaglianza di cui l'altro soffre. 
La violabilità dei diritti non è uno scenario desiderabile per chiunque: nessuno vorrebbe vedere violati i propri diritti e questa tensione comune rivendica un fondamento più solido della libertà del soggetto, rintracciando la consapevolezza che la dignità della persona deve essere uguale per tutti. Di conseguenza proclamare un diritto umano coinvolge il riconoscimento della persona nella sua autonomia ma anche nella sua necessaria relazione con il suo fine e con la dignità dell'altro.

Va tenuto conto, poi, che non tutti i diritti hanno il loro fondamento nella persona allo stesso modo. Alcuni precedono qualsiasi legge, perché toccano la radice stessa della persona, che trascende lo stesso ordinamento giuridico: il diritto alla vita e il diritto del nascituro di vedere rispettata la propria vita. Altri sono legati al tempo e alle circostanze, e sono frutto della determinazione ulteriore della legge (SEN, 2010, p.369) $)^{5}$. Esistono pertanto dei diritti umani assoluti, nel senso che si fondano in modo immediato sulla stessa dignità della persona e che quindi non possono mai essere cancellati o violati, senza offendere la stessa dignità della persona umana. In nome di questi diritti l'art. 33 della Convenzione di Ginevra del 28 luglio 1951 impone gli Stati contraenti a non respingere gli immigrati verso le frontiere laddove rischiano la persecuzione per motivi di razza, religione, nazionalità, appartenenza ad un gruppo sociale, opinione politica.

\section{IL RUOLO DEI DIRITTI UMANI NELLA PROTEZIONE DEI MIGRANTI}

Norberto Bobbio nella sua opera L'Età dei diritti ribadisce molte volte che il problema più urgente oggi, relativo ai diritti dell'uomo, non è tanto quello di "giustificarli", quanto quello di "proteggerli". Non si tratta tanto di sapere quali e quanti sono i diritti dell'uomo, quali sia la loro natura,

\footnotetext{
${ }^{5}$ Vedi n. 2. Se per il fondatore dell'utilitarismo moderno, Jeremy Bentham, i diritti umani sono "figli della legge" (per essere qualificabile come un diritto una rivendicazione deve avere una forza legale e ogni altro uso del termine "diritto" è sbagliato), per Herbert Hart i diritti umani sono in realtà "genitori della legge", giacché possono esserne la fonte d'ispirazione. Per Amartya Sen, Hart ha assolutamente ragione: non ci sono dubbi che la nozione di diritti morali possa fungere da stimolo per lelaborazione di nuove norme giuridiche. Oltre a stimolare l'azione legislativa, il riconoscimento dei diritti umani può trovare molte altre applicazioni. Grazie all'importanza di elementi come la comunicazione, le pressioni, le denunce e un dibattito pubblico ben informato, i diritti umani possono esercitare una certa influenza senza dipendere necessariamente da norme di legge coercitive.
}

bensì quale sia il modo più sicuro per garantirli, per impedire che, nonostante le dichiarazioni solenni, vengano continuamente violati. Cercare il fondamento etico dei diritti dell'uomo è certamente un'impresa difficile e legittima, ma non avrà alcuna importanza storica se non sarà accompagnata dallo studio delle condizioni, dei mezzi e delle situazioni in cui questo o quel diritto possa essere realizzato. Pertanto la questione del fondamento etico dei diritti dell'uomo non può essere dissociato dallo studio dei problemi storici, sociali, economici, psicologici inerenti alla loro attuazione (BOBBIO, 1990, p.16). In questa sede cercheremo di capire se i diritti umani, validi sempre, dappertutto e per tutti, costituiscono di per sé una piattaforma giuridica idonea a garantire una protezione fondamentale dei migranti. Difatti i diritti umani appaiono congruenti con il perseguimento di una tutela ultra-territoriale dei cittadini ed ultra-civica degli stranieri: essi intessono di per sé la tela di un diritto trans-nazionale.

La fine della seconda guerra mondiale, con gli orrori che ha recato con sé la Storia, ha decretato, tra i molteplici insegnamenti seminati, la convinzione che le persone non devono essere torturate, un principio imprescindibile che è cresciuto esponenzialmente nel mondo. L'art. 5 della Dichiarazione universale dei diritti dell'uomo, recita: "nessun individuo potrà essere sottoposto a tortura o a trattamento o a punizione crudeli, inumani o degradanti”. Questo divieto è stato ripreso in molti altri documenti come si legge nell'art. 3 della Convenzione europea per la salvaguardia dei diritti dell'uomo e delle libertà fondamentali del 4 novembre 1950: "nessuno può essere sottoposto a tortura né a pene o trattamenti inumani o degradanti".

Il divieto di tortura lo troviamo espresso, indirettamente, sottotraccia, nella Convenzione di Ginevra del 28 luglio 1951, con successivo Protocollo adottato a New York il 31 gennaio 1967 (trattati resi esecutivi in Italia, rispettivamente con legge n. 722 del 24 luglio 1954 e con la legge n. 95 del 28 marzo 1970) che individua e descrive la condizione di rifugiato riconoscendola in tutti i casi in cui un uomo sia portatore del giustificato timore di essere vessato per la sua razza, religione, cittadinanza, appartenenza a un determinato gruppo sociale, o per opinioni politiche, che si trovi fuori dallo Stato di cui possiede la cittadinanza e, spinto dalla paura di essere perseguitato, non può - o non vuole - domandare la protezione di detto Stato. 
La Convenzione non riconosce come rifugiato le persone sospettate - con ragionevoli motivi - di avere commesso: un crimine contro la pace, un crimine di guerra o un crimine contro l'umanità; un reato grave del diritto comune fuori dal Paese ospitante prima di essere ammesso come rifugiato. Per esempio, un cittadino straniero che è imputato di un delitto comune (omicidio durante una rissa) punito nel paese di origine con la pena di morte non ha diritto al riconoscimento dello status di rifugiato politico (art. 7 d. lgs. 19 novembre 2007, n. 251). Non è pure riconosciuto come un rifugiato politico la persona che ha commesso reato non grave ma punibile con la reclusione nello Stato di rifugio, laddove 1'allontanamento sia strumentale ad evitare la sanzione; infine la Convenzione di Ginevra non riconosce come rifugiato la persona sospettata di commettere atti contrari agli scopi e ai principi delle Nazioni Unite, nonché rappresenti un pericolo per la comunità o la sicurezza dello Stato in cui si trova.

Ai sensi dell'art. 33 della Convenzione è fatto divieto agli Stati di espellere o respingere i rifugiati e i richiedenti asilo verso i luoghi in cui la loro vita o la loro libertà ne sarebbero minacciate per motivo di razza, di religione, di cittadinanza, di appartenenza a un gruppo sociale o per la loro opinione politica (PETROVIĆ, 2011, p.23; BUONOMO, 2013, p.96; RITA GIL, 2017). È il cosiddetto principio di nonrefoulement. Questo principio trova applicazione anche nel caso in cui l'espulsione avvenga verso un Paese definito di transito o intermedio, che potrebbe a sua volta rinviare la persona in un territorio in cui sarebbe esposta alla persecuzione per motivi sopra evidenziati. L'obbligo di non-refoulement è un limite assoluto, non derogabile e non bilanciabile nemmeno con ragioni di sicurezza nazionale e di contrasto al terrorismo internazionale. Rimpatriare chi rischia di subire gravi violazioni dei diritti umani o persecuzioni nel paese di origine costituisce una violazione del principio di non-refoulement, a prescindere dal fatto che questi abbia potuto attivare la procedura per la concessione dell'asilo politico e persino nell'ipotesi che abbia avuto esito negativo.

L'espulsione a titolo di misura di sicurezza secondo l'art. 3 della Convenzione europea per la salvaguardia dei diritti dell'uomo, sopra preannunciato e gli artt. 2 lett. g, e 20 del d. lgs. n. 251 del 2007, non può essere eseguita lì dove esponga il soggetto a pericoli per la incolumità o a trattamenti disumani e degradanti, per motivo razza, di sesso, di cittadinanza, di religione, di opinione politica, di condizioni personali o possa rischiare di essere rinviato verso un altro Stato nel quale non sia protetto dalla persecuzione. Oltre alla condizione di rifugiato tracciata nel testo della Convenzione di Ginevra, la direttiva n. 83 del Consiglio Europeo del 2004 (2004/83/CE del Consiglio) prevede che il cittadino del Paese terzo che non possiede i requisiti per essere riconosciuto come rifugiato ma nei quali confronti sussistono fondati motivi per ritenere che, se tornasse nel paese di origine correrebbe un rischio effettivo di subire la condanna alla pena di morte, tortura o altra forma di trattamento inumano e degradante, e il quale non può o a causa di tale rischio non vuole avvalersi della protezione di detto Paese, non può essere espulso.

In questo senso, emblematico è il caso Khemais del $2009^{6}$ (RUGGIU, 2009, p. 666), cittadino tunisino condannato a dieci anni per terrorismo. Fuggito in Italia, si era macchiato del reato di associazione a delinquere, per cui lo raggiunse un decreto di espulsione. Invocando i rapporti di Amnesty International e Human Rights Watch, Khemais si appellò alla Corte di Strasburgo chiedendo il suo intervento per rimanere in Italia e non essere rimpatriato perché avrebbe potuto subire torture o trattamenti inumani e degradanti in Tunisia. La Corte, non ignorando tale possibilità, chiese alle autorità italiane di sospendere l'esecuzione del decreto di espulsione ${ }^{7}$. La sentenza dimostra come il divieto di tortura rappresenti un limite insormontabile anche quando la sicurezza dello Stato appare seriamente minacciata. In questi casi lo Stato ha l'obbligo di adottare misure di sicurezza tipologicamente diverse dall'espulsione.

Sull'argomento, il Rappresentante speciale delle Nazioni Unite sulla tortura, Nils Melzer, ha presentato il 26 febbraio 2018 un dossier in cui vengono segnalate politiche sull'immigrazione che non tengono sufficientemente conto del rischio di tortura e dei trattamenti disumani (MELZER, 2018). Secondo Melzer i diversi strumenti messi in campo da numerosi Stati dell'Unione europea hanno come obiettivo precipuo il blocco degli arrivi e la difesa del

\footnotetext{
${ }^{6}$ Sentenza della Corte europea dei diritti dell'uomo, Khemais et autres c. Italie (requêt 246/07), arrêt 24 février 2009.

${ }^{7}$ Va tenuto presente che per portare un caso alla Corte europea dei diritti dell'uomo il ricorrente dovrà prima esaurire tutti i rimedi previsti dalla legge del paese di appartenenza. Lappello alla Corte di Strasburgo non elimina il rischio d'espulsione, per cui la decisione della Corte potrebbe risultare tardiva e incapace di produrre alcun effetto pratico.
} 
benessere degli Stati che non deve essere intaccato dall'onda migratoria. Vanno in questa direzione gli Accordi internazionali e le scelte politiche che hanno come obiettivo centrale semplicemente bloccare gli arrivi di migranti, costi quel che costi. Al di là delle apparenze formali instaurate attraverso accordi bilaterali internazionali, quello che viene messo in atto è una violazione dei diritti umani.

Fa notare Marco Borraccetti: "le scarse possibilità di ingresso regolare rischiano di incentivare [anzi incentivano] il ricorso a vie di accesso irregolari, che loro volta inducono l'adozione di ineficaci politiche di prevenzione e contrasto di immigrazione irregolare" (BORRACCETTI, 2016, p.33). L'emanazione di leggi e pratiche che spingono i migranti a utilizzare rotte e metodi irregolari, contribuisce alla diffusione di pratiche di tortura e di trattamenti disumani e degradanti, e in questo senso non si limita a una violazione dell'art. 33 della Convenzione di Ginevra del 1951 (principio di non-refoulement) e dell'art. 3 della Convenzione europea dei diritti dell'uomo del 1950, ma il dettato legislativo incorre nel reato di complicità in crimini contro l'umanità.

Nello stesso giorno in cui Nils Melzer pubblicava il dossier sulle politiche migratorie che non esulano dal rischio di tortura e trattamenti disumani e degradanti, il consiglio d'Europa, tramite il Rappresentante speciale del Segretario generale per le migrazioni e i rifugiati Tomáš Boček rendeva noto il primo rapporto sulle risposte degli Stati ai flussi migratori (BOČEK, 2018). Tomáš Boček evidenziava come il rafforzamento dei controlli alle frontiere di numerosi Stati membri dell'Unione europea riduceva gli arrivi in Europa, ma a scapito dell'effettivo rispetto dei diritti umani. Così il Rappresentante speciale del Segretario generale per le migrazioni e i rifugiati ammoniva severamente gli Stati che perseguono politiche e pratiche in materia di immigrazione ignorando i diritti umani.

\section{CONCLUSIONE}

È innegabile che il principio di sovranità implica il diritto dello Stato di controllare le frontiere e di definire le procedure per l'ammissione degli stranieri sul territorio, ed è altrettanto innegabile che in una società liberaldemocratica - per principio - queste prerogative statuali sono temperate e limitate dai diritti umani, di cui gli individui beneficiano non in quanto cittadini, bensì come persone. Si tratta di quello che il sociologo italiano Maurizio Ambrosini definisce "il vincolo liberale (liberal constraint)" (AMBROSINI, 2017, p. 60). Il vincolo liberale ostacola la capacità dei tradizionali Paesi di immigrazione di perseguire efficacemente l'immigrazione irregolare: "impedisce di attuare provvedimenti drastici di deportazione, ricorso alle armi per fermare chi attraversa illegalmente i confini, espulsione di massa senza garanzie giuridiche, irruzioni in abitazioni private alla ricerca di immigrati irregolari"'( AMBROSINI, 2017, p. 60). Seguire una strada di questo tipo - di repressione del passaggio non autorizzato delle frontiere o del soggiorno del soggetto non autorizzato nel territorio - comporterebbe per le democrazie (occidentali) il rischio di cadere in contraddizioni pericolose per la loro stessa natura. Se le democrazie liberali vogliono essere coerenti devono dimostrare che i modi con cui vigilano i propri confini non violano i diritti umani fondamentali.

È innegabile che oggi è sempre più diffusa, sia a livello individuale che collettivo, un' attenzione crescente alla sussistenza di diritti umani inviolabili, che vanno pertanto adeguatamente garantiti. Paradossalmente, oggi, mentre cresce la sensibilità e il favore per i diritti umani, si assiste al singolare fenomeno per cui forse mai, come nel nostro tempo, si sono perpetrati attentati contro i diritti umani di ogni sorta: dalle sanguinose guerre di aggressione alle altrettanto sanguinose guerre civili, dai genocidi programmatici alle sinistre operazioni di pulizia etnica. È evidente, in questo contesto, come il ruolo dei diritti umani nella protezione degli immigranti sia assolutamente marginale.

Uno dei elemento caraterristico che determina la debolezza dei diritti umani nella protezione degli immigranti concerne la difficoltà per "le vittime" di accedere al sistema di protezione da essi delineato. L'immigrato in situazione irregolare, al suo approdo nel paese di immigrazione, non ha null'altro che la propria "vita nuda" (AGAMBEN, 1995), come definita da Giorgio Agamben. Non può contare sulla protezione del paese di arrivo, ma può soltanto fare appello all'inalienabilità dei diritti umani. Nonostante ciò, la chiamata degli immigrati irregolari viene disattesa perché i migranti non riescono ad accedere al sistema di protezione dei diritti umani, temendo di essere rimpatriati se presentano il loro caso all'autorità del governo del paese dove soggiornano irregolarmente. Pertanto, non è del tutto sbagliata la ricorrente impressione che il sistema dei diritti umani 
protegga coloro che sono già protetti: infatti, i diritti umani si basano sì sulla nuda vita, sull'idea costitutiva di appartenenza alla famiglia umana, ma il loro pieno esercizio dipende dall'essere soggetto di uno Stato, membro legalmente residente di una nazione.

\section{BIBLIOGRAFIA}

AGAMBEN, G. Homo sacer. Il potere sovrano e la nuda vita, Einaudi, Torino 1995.

AMBROSINI, M. Migrazioni, Egea, Milano 2017.

ALEXY, R. Diritti umani senza metafisica? In «RIFD Rivista Internazionale di Filosofia del Diritto», Giuffrè Editore, serie V - anno XCII - 2015.

BOBBIO, N. L'età dei diritti, Einaudi, Torino 1990.

BORRACCETTI, M. L'Italia e I rimpatri: breve ricognizione degli accordi di riammissione, in «Diritto, Immigrazione e cittadinanza», XVIII, 2016.

BOČEK, T. First report on the activities of the Secretary General's Special Rapresentative on Migration and rifugees, 1 february 2016 to 31 january 2018. Disponibile in http://www.marinacastellaneta.it/blog/wp-content/ uploads/2018/03/SRSG-Activity-Report.pdf. Accesso 11 mar. 18.

BUONOMO, V. L'efficacia e limiti del Diritto internazionale in tema di mobilità umana. Alla ricerca di un nuovo paradigma, in «Apollinare», LXXXVI (2013).

DE PAOLIS, V. Diritti umani e fondamento etico, in G. BATTISTELLA (a cura di), «Migrazioni e diritti umani». Quaderni SIMI 1, Urbaniana University Press, Città del Vaticano - Roma 2004.

FERRAJOLI, L. Manifesto per l'uguaglianza, Laterza, BariRoma 2018.

FRANCESCHINI, A. Oltre la crisi della relazione affettiva. Il personalismo etico di Karol Wojtyla, Tau Editrice, Todi (PG) 2012.

HABERMAS, J. Il concetto di dignità umana e l'utopia realistica dei diritti umani, in J. HABERMAS, «Questa Europa è in crisi», tr. it. di C. Mainoldi, Laterza, Roma-Bari 2012

HABERMAS, J. Fatti e norme. Contributi a una teoria discorsiva del diritto e della democrazia, a cura di L. Ceppa, Laterza, Roma-Bari 2013.

HABERMAS, J. Etica del discorso, a cura di E. Agazzi, Laterza, Roma-Bari 1989.

HEGEL, F. W. G. Fenomenologia dello spirito, Bompiani, Milano 2000.
HOBBES, T. Leviatano, tr. it. A. Lupoli, Roma-Bari, Laterza 1989.

JOSÈ ERRÀZURIZ, C. (et al), Realismo e diritto naturale. Un dibattito su cos'è il diritto? Di Javier Hervada, in «Rivista Internazionale di Filosofia del Diritto», Serie VAnno XCIIV 2-3, 2017.

MARCHETTONI,L. Habermas sui diritti umani. Dalla validità discorsiva alla dignità umana, in «Jura Gentium Rivista di filosofia del diritto internazionale e della politica globale», 2, 2012.

MELZER, N. Report of Special Rapporteur on torture and other cruel, inhuman or degrading Treatment or punishement, 26 febbraio 2018. Disponibile in http://www. marinacastellaneta.it/blog/wp-content/uploads/2018/03/A_ HRC_37_50_EN.pdf. Accesso 11 mar. 18.

MURA, G.L'eticaelapoliticadell'alterità: Multiculturalismo e riconoscimento in G. Battistella (a cura di), «Migrazioni. Questioni etiche», Quaderni SIMI 6. Urbaniana University Press, Città del Vaticano - Roma 2008.

PEROTTI, A. Diritti alla diversità culturale e diritti alla somiglianza universale: componenti individuali dei diritti umani, in G. BATTISTELLA (a cura di), «Migrazioni e diritti umani», Quaderni SIMI 1, Urbaniana University Press, Città del Vaticano - Roma 2004.

PETROVIĆ, N. Rifugiati, profughi, sfollati. Breve storia del diritto d'asilo in Italia dalla Costituzione ad oggi, FrancoAngeli, Milano 2011.

PETRUCCIANI, S. Introduzione, in G. CALOGERO, Filosofia del dialogo, Morcelliana, Brescia 2015.

PONTIFICIO CONSIGLIO DELLA GIUSTIZIA E DELLA PACE, Compendio della dottrina sociale della Chiesa (CDSC), Libreria Editrice Vaticana, Città del Vaticano 2004.

RICOEUR, P. Percorsi del riconoscimento. Tre studi, a cura di F. Polidori, Cortina, Milano, 2005.

RITA GIL, A. Imgração e direitos Humanos, Petrony Editora, Lisboa 2017.

ROBINSON, M. I diritti dell'uomo, storia e speranza, in Coscienza e libertà, n. 52, 2016.

RUGGIU, G. Il concetto della libertà di movimento in Europa. Un'analisi delle norme convenzionali e della giurisprudenza della Corte di Strasburgo, in «Diritto e questioni pubbliche», n. 9, 2009.

SEN, A. L'idea della giustizia, tr. it. di Luca Vanni, Mondadori, Milano, 2010.

SCHINO, L. A. La teoria hobbesiana delle passioni: psicologia materialistica e ricerca della felicità in C. Borghero e A. Del Prete (a cura di) «L'uomo, il filosofo, le passioni», Le lettere, Firenze 2016. 